\title{
Exploring Dynamic Character Motion Techniques for Games Development
}

\author{
Ismahafezi Ismail, Mohd Shahrizal Sunar, Cik Suhaimi Yusof
}

UTM ViCubeLab, Department of Computer Graphics and Multimedia, Faculty of Computer Science and Information Systems, Universiti Teknologi Malaysia.

\begin{abstract}
D character development is very important part in the character animation. Currently, animation researchers try to control their virtual character joint and make their character motion more realistic and look like real human movement. Using motion capture technology, input data for character movement can be manipulated. This paper presents a current motion research in the real time animation character and focused in dynamic motion control considering physic for game development. From this paper, the researcher can get better understanding what is the main issues and relevant technique that used by the recent researchers in this area. This review focuses on three main parts in dynamic motion generation with physics consideration and control: skeleton hierarchy and kinematics, motion capture data animation, and active dynamic control.
\end{abstract}

Index Terms - Motion Capture, Realistic Motion Data, 3D Character Animation.

\section{INTRODUCTION}

Natural character movement is the main issue in 3D character animation area. The development involves character skeleton joints and bones that will simulate movement through virtual environment. Using 3D Software, animator will design the character and make a model optimization. After that, skeleton will be set up for character rigging. The main process is to edit input data for mapping into the character before produce the movement animation. Fig. 1 shows 3D character development process. Generally, the movement of a character in computer games is not smooth and realistic when compared with the movement of people resulting to the non-real-time animation as cartoons and movies. To achieve the realistic virtual character, a character should be adjusted so that a movement similar to humans in the real world. Analysis of actual human movement data can be applied to characters in real time using motion capture technology. One of the biggest challenges in interactive computer games industry is to produce a dynamic character movement and a reaction to the physical interaction.

Unlike real human, motions created by the animators are fixed. These motions are created to fit certain situations of motion and their connections are created. As a result, the character will be repeating the same motion in real time animation. Therefore, it looks impractical and the character

Manuscript received on May 11, 2011

E-mail: ismahafezi@yahoo.com. cannot respond to physical interaction with other autonomous characters. Character in real time animation requires a combination of motions from different sources such as motion capture, manual keyframes, dynamic and kinematics simulation [1]. A summary of this approach is illustrated in Fig. 2.

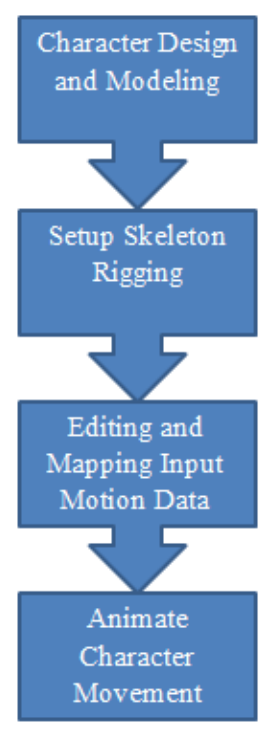

Fig. 1. 3D Character development

Dynamic motion refers to the physical properties of 3D object, such as mass or inertia, and specifies how the external and internal forces interact with the object [2]. With the dynamic of character data, the control of the character's specific motion: walking, running and jumping looks more realistic. Real-time characters consist of solid sections connect by joints. These sections and joints are known as a skeleton. Motion of the skeleton can be specified in terms of rotation and translation. The kinematic approach refers to virtual character properties such as position, orientation, and velocity. Forward kinematics approach directly controls the relative geometric transformation of each bone of the skeleton meanwhile Inverse Kinematics is about solution of geometric locations to a given point.

In this paper, we analysis the basics of hierarchy in producing dynamic motion control considering physic for real time animation character. This review will concentrate on skeleton hierarchy and kinematics, motion tracking data animation, and active dynamic control. 


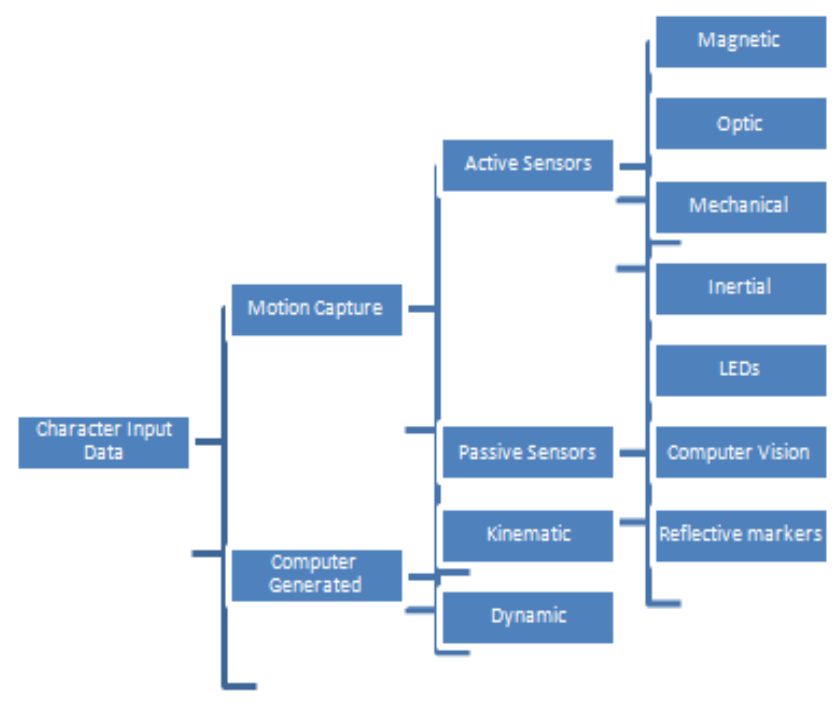

Fig. 2. Sources for the character motion in real time animation

\section{SKELETON HIERARACHY AND KINEMATICS}

Generally, human movement development in computer games involves joints of 3D models controlled by the skeleton hierarchy. These joints have been combined with three-dimensional geometric models, such as polygonal mesh. There are three main techniques commonly used to produce animation for the characters as shows in Fig. 3.

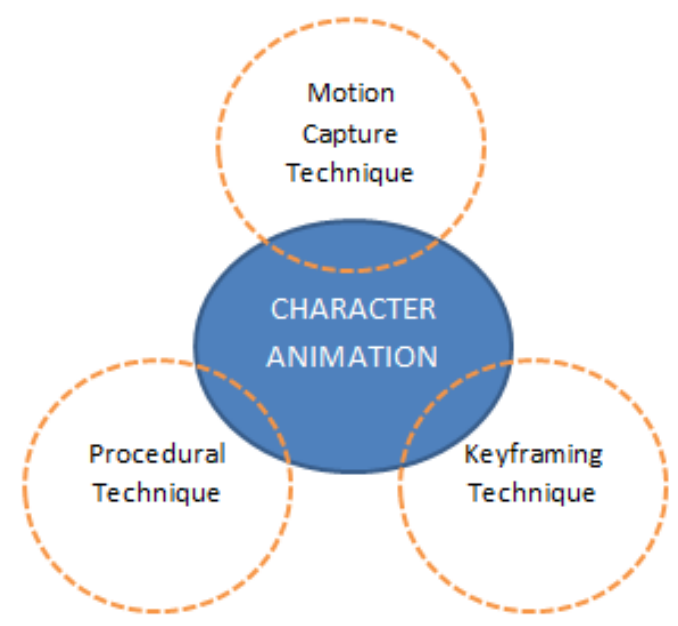

Fig. 3. Character animation techniques

Key-framing technique involves main frames for main character poses and in-between frames that used to transform the position, placing, or timing of an object. The animator will make character movement editing and interpolation at in-between frames and has direct control over the positions, shapes, and motions of character at any moment in the animation. The effect of changing a parameter value is often predictable in keyframing techniques. Meanwhile, procedural technique provides initial conditions and adjusts rather abstract physical parameters, such as forces and torques. The animator has to run a simulation in virtual environment to see the result of parameter value changing. The animator specifies physical rules and initial conditions to the character. The simulation calculation will be used kinematic or dynamic methods.

Currently, motion capture techniques have been used widely from video game animation to computer graphic effects in movies. An actor, are placed in a special suit containing sensors that record the motion to get the real human movement data. The motion data output is often far from perfect because need to clean up from keyframe animator to make it look more natural. The output data from motion capture technology shows more realistic, convincing and better character movement than other techniques [27].

In general, human character in real animation was represented by a skeleton. A skeleton is described by the arrangement of bones that have been linked by specific joints [4]. A real time animation character has 16 rigid links and 23 main ball joints that can be manipulate and control as shown in Fig.e 4. The total number of degrees of freedom (DOF) of virtual character is $41[5]$.

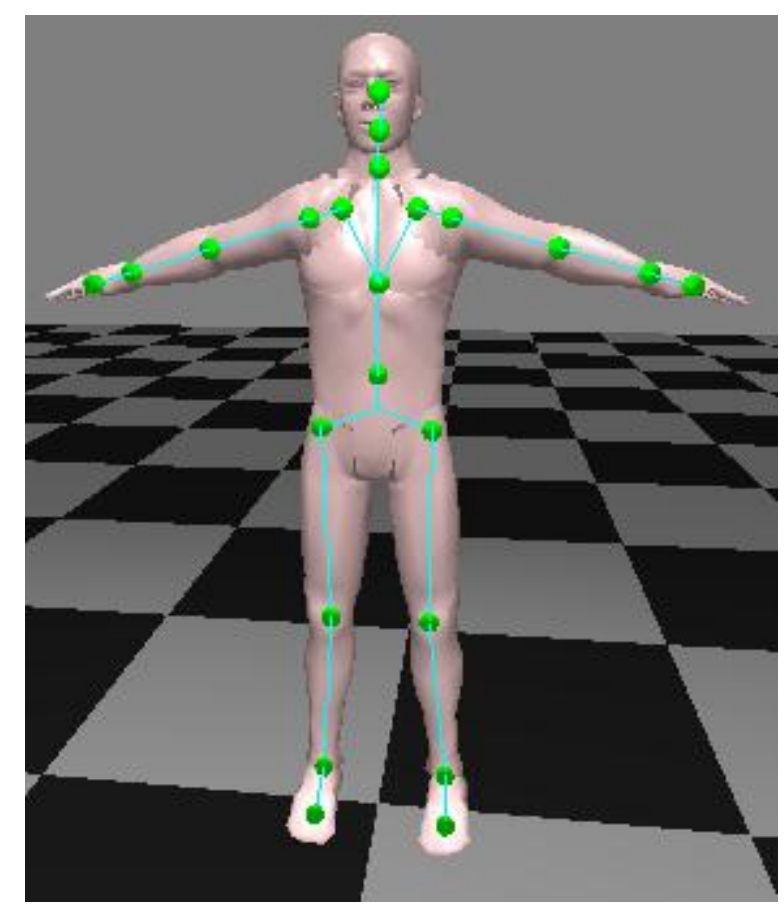

Fig. 4. Human figure model with skeleton joints (Color Plate 10)

3D character movement controlled using skeleton structure or hierarchy. Example of 3D character motion data hierarchy shows in Fig. 5. A hierarchy uses grouping or parenting concept. For example, of human leg, the hip is the parent of the upper leg. Meanwhile, the lower leg is the child the upper leg, and the foot is the child of the lower leg. In real time animation environment, each bone depends on the orientation and the joint with its parent [4]. Generated motion has to enforce with physical law of motion for creating the realistic human character motion. 


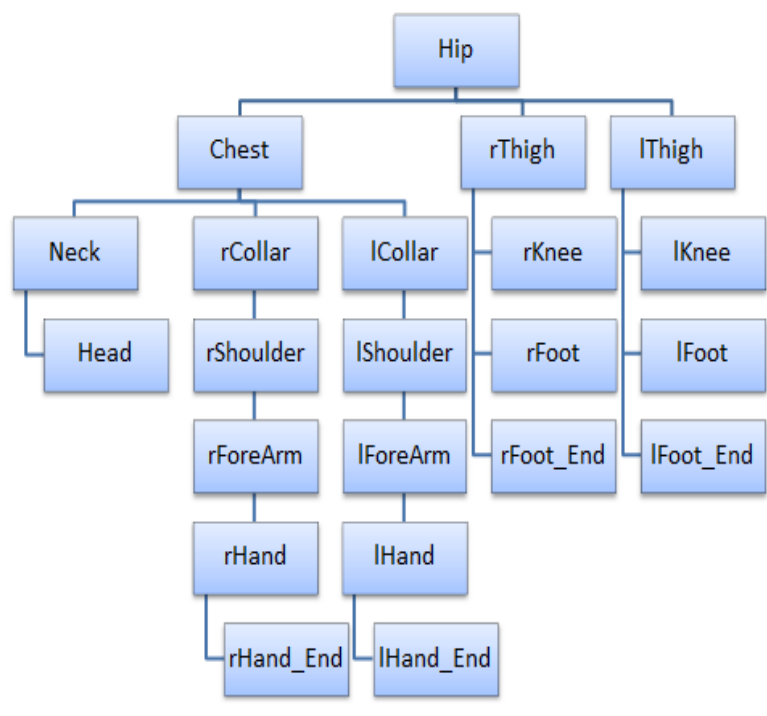

Fig. 5. Character Motion Data Hierarchy

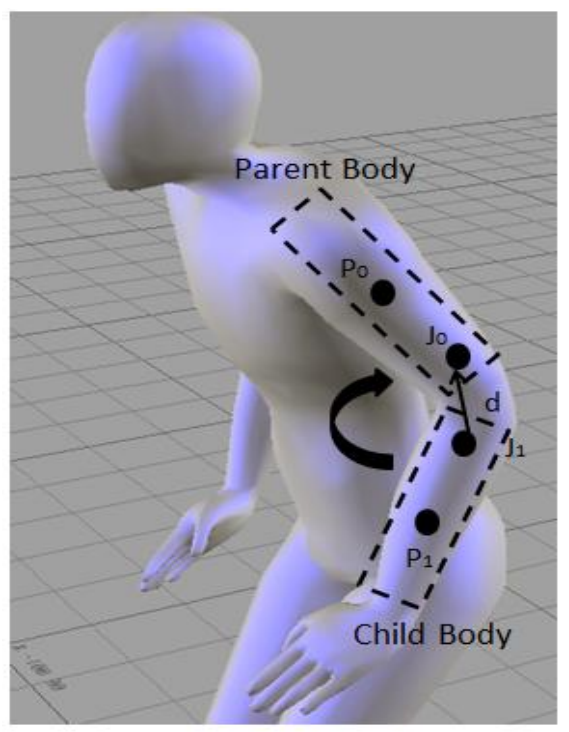

Fig. 6. Examples of character parent-child body relationship

Fig. 6 shows parent body and required translation to the child body for 3D character. After transform the child body into global space, we can calculate the new position of the body. The parent body rotation matrix defined as $R_{0}$ and parent body position as $\vec{p}_{0}$. When the parent body move, automatically the child body transform from local body space to global space. The parent body position transformation:

$$
\vec{p}^{\prime}{ }_{o}=R_{0} \vec{l}+\vec{p}_{o}
$$

Meanwhile, the child body is defined as $\mathrm{R}_{1}$ and $\vec{p}_{1}$. When the parent body moves, the joint $J_{0}$ and $J_{I}$ change to the same coordinate place. To transform child body from local space into global space:

$$
\vec{j}_{n}^{\prime}=R_{n} \vec{j}_{n}+\vec{p}_{n}, \mathrm{n}=0,1
$$

To get the vector $\vec{d}$, we subtract $\vec{j}_{0}$ and $\vec{j}_{1}$. The new body position, $\vec{p}_{1}^{\prime}$ :

$$
\vec{p}^{\prime}{ }_{1}=\vec{p}_{o}+R_{o j} \vec{j}_{o}-\vec{R}_{1} j_{1}
$$

The function of inverse kinematics and forward kinematics is to calculate the bone's position, including the joints position and angles [6]. Normally, inverse kinematics is used for motions involving the lower part of a character's body such as joints from the foot to the pelvis $[4,5,6]$. It is very hard to use forward kinematics because the body position will be moving below the surface or ground. This transaction makes the character's motion very unreliable and unconvincing.

In the case of shoulder rotation, or to get the position of the upper arm, we use forward kinematics calculation [6]. Forward kinematics is a top-down technique rotation used to position the character's upper body part in real time animation. Each skeleton joint has its local transformation, and parent transformation will determine the global transformation of each skeleton joint.

$$
\mathrm{M}_{\text {global }}^{\mathrm{n}}=\Pi_{\mathrm{i}=0}^{\mathrm{n}} \mathrm{M}_{\text {local }}^{\mathrm{i}}
$$

$n$ here represents the current joint in the hierarchy.

In summary, forward kinematics can control the rotation of a skeleton. Single joints and the children joints will follow automatically when we rotate top level joints. On the other hand, inverse kinematics is useful when we need to move a single object to pose in the above direction of the skeleton hierarchy from the moving joint.

\section{MOTION CAPTURE DATA ANIMATION}

Motion capture technology has been used in many areas, including computer games character, character motion research and movie making. Using motion capture devices, a researcher can manipulate and track full body motion of the character in the virtual world. The purpose of motion tracking is to get a sample of frames that can replace the motions of real human in the virtual environment. Motion tracking records the position of each joint of the human skeleton [7]. With the motion data, reliable character motions can be created in real time animation. Motion capture technologies are generally classified into active and passive sensor-based capture according to the nature of the sensor used [3, 19]. This sensor can mimic a real human movement, and the raw measurement data will be applied to the virtual character motion.

Editing and reuse of motion capture data have become a challenging task to the researcher. The early framework introduces optimal trajectory method [8] that solved dynamic constraint problem. A famous methodology for producing a natural character motion in real time animation is the space time optimization framework $[9,10,20]$. We can edit or use motion capture data and keep physical properties at the same 
time using this method. Recently, there have been many studies on motion capture data editing and retrieval. One of them utilized fuzzy inference to create a personalized animation of virtual character [11,23]. This method uses fuzzy rules to calculate motion capture data parameters. Algorithm for fuzzy rules is created from the results of subjective animated movement. New proportionality coefficients $\alpha$ and $\beta$ was defined that are strongly correlated with subjective features of animation [11]. A comparison of this approach is illustrated in Table 1.

To modify same motion capture data, dimension of the data needed to be reduced using principal component analysis (PCA), making a trained dictionary to get indexed motion data, and test the data using support vector machine (SVM). This method can reduce the retrieval time of motion capture data [12]. PCA can reduce the dimensions of motion capture data, which is compressing and matching the input parameter [13]. The outline of this method has two core parts: process of making dictionary and process of posing discrimination. From the input training data, the output training dictionary processed with SVM to get the retrieval data. A summary of input and output motion capture data for retrieval process is illustrated in Fig. 7.

TABLE 1: COMPARISON OF MOTION CAPTURE DATA EDITING TECHNIQUE

\begin{tabular}{|l|l|}
\hline Technique & Description \\
\hline & $\begin{array}{l}\text { These technique involved combination } \\
\text { between motion capture data and } \\
\text { dynamic simulation [9, 14, 15] to } \\
\text { develop character motion while interact } \\
\text { with virtual environment. The editing } \\
\text { process using correction of the } \\
\text { motion editing } \\
\text { momedum and balance constraints. }\end{array}$ \\
\hline $\begin{array}{l}\text { Interpolation- } \\
\text { based motion } \\
\text { editing }\end{array}$ & $\begin{array}{l}\text { Straightforward interpolation of joint } \\
\text { angles [14] and blend motion sequences } \\
\text { with various inverse kinematic [16]. }\end{array}$ \\
\hline $\begin{array}{l}\text { Momentum-b } \\
\text { ased motion } \\
\text { editing }\end{array}$ & $\begin{array}{l}\text { Optimize the algorithm from motion } \\
\text { capture data to meet high-level user } \\
\text { limitation [14] while protecting physical } \\
\text { properties. }\end{array}$ \\
\hline $\begin{array}{l}\text { Avatar fuzzy } \\
\text { inference }\end{array}$ & $\begin{array}{l}\text { Algorithm for fuzzy rules has been } \\
\text { created from results of subjective } \\
\text { animated monement. } \\
\text { proportionality coefficients } \alpha \text { and } \beta \text { was } \\
\text { defined that are strongly correlated with } \\
\text { subjective features of animation [11]. }\end{array}$ \\
\hline $\begin{array}{l}\text { Interaction } \\
\text { mesh }\end{array}$ & $\begin{array}{l}\text { New approach for editing motions that } \\
\text { involve close interactions such as } \\
\text { dancing, fighting and other motions } \\
\text { [17]. }\end{array}$ \\
\hline
\end{tabular}

Motion tracking is the core part in character motion dynamic and control. Using motion data, a user can directly control and modify the character motion in real time animation.

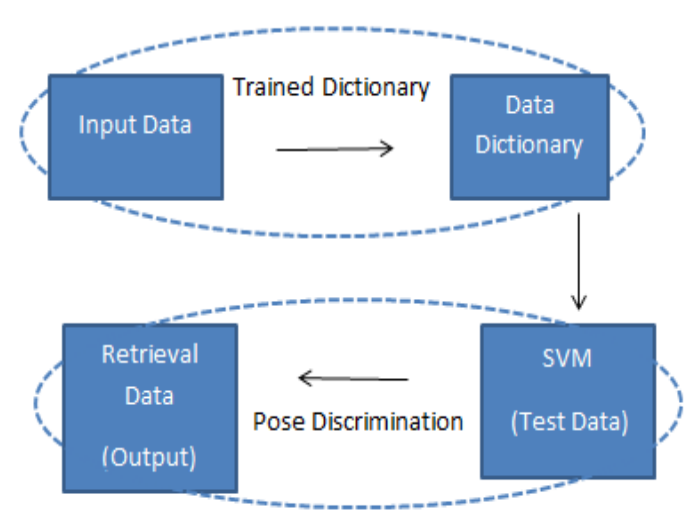

Fig. 7. Summary of input and output of motion capture data

\section{ACTIVE DYNAMIC CONTROL}

3D games character has a rigid body that has its own forces, velocity, mass and physical properties. The main step to control dynamic motion simulation is the basic dynamics of that character's movement. Using dynamic engine application such as havoc physic, massive and open dynamic engine (ODE), the physical realistic of real time animation has been upgraded to another level. This application can solve dynamic calculation $[22,25,27]$, dynamic contact and friction for the rigid body dynamic.

Although physic properties can be applied to character motions in real time animation, however it is still limited when it comes to rigid objects. Active dynamic control can be achieved from any athlete motion [4] for example, cycling, fighting, running and swimming. A feedback control system [4, 24] was used to process motion capture data. Human motions can be generated using physical simulation such as a robotic-derived controller based approach and performance optimization based approach [18].

A lot of researcher focuses on study of dynamic character as shows in Fig. 8. Related research about dynamic motion control:

\subsection{Motion Control Based on Dynami}

A lot of approaches have been developed for the purpose of character motion control based on dynamic. The example given, using a muscle strength model [21] for the inverse kinematics method. This method can calculate and change the motion speed and trajectory using the joints of skeleton [26]. Another technique is creating motion using inverse kinematics method and produces a human walking motion using inverse dynamic [26]. 


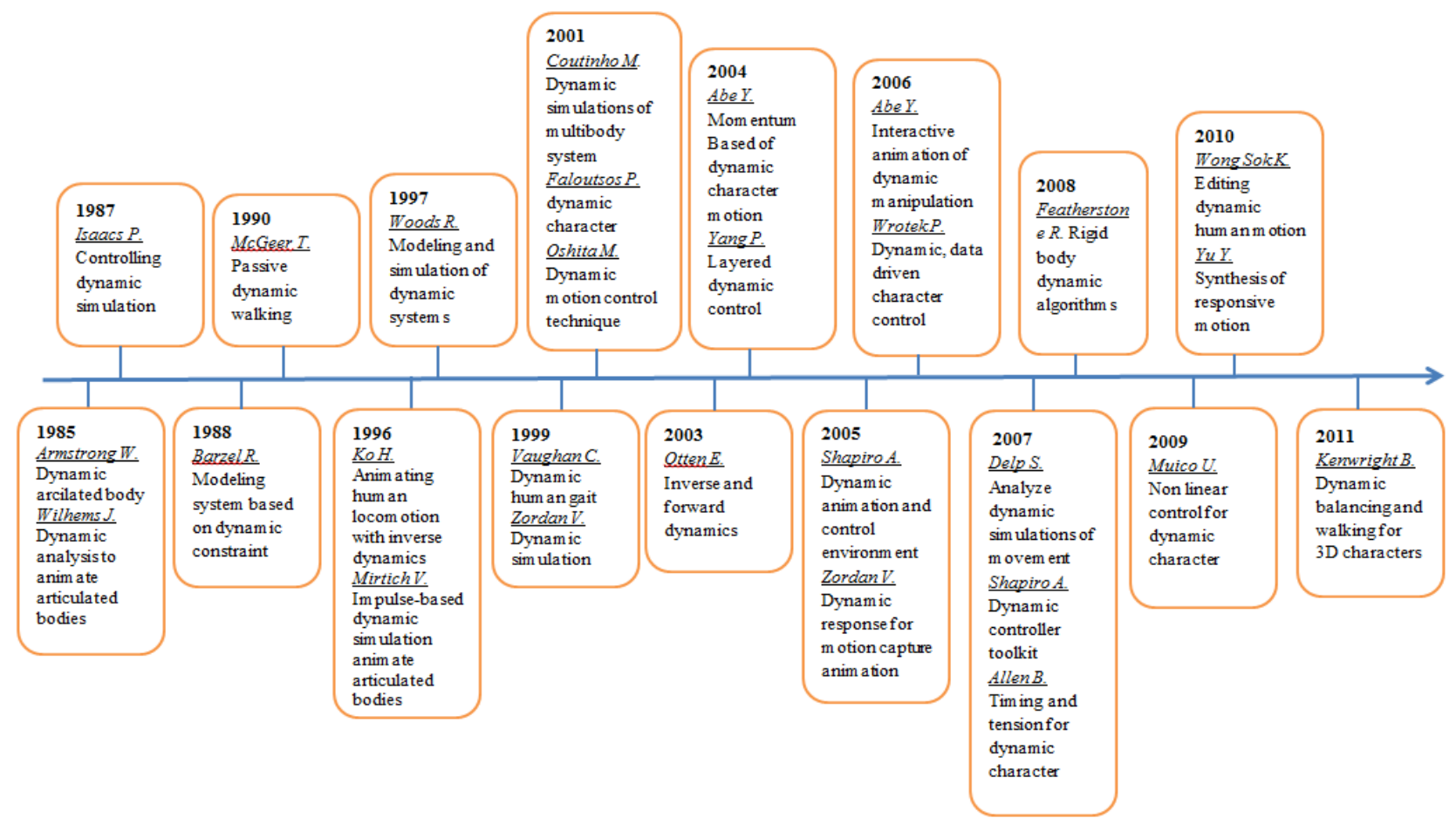

Fig. 8. Research focus on dynamic motion character area

\subsection{Structure of dynamic motion control}

In general, dynamic motion control must have two core parts: controller and simulator. The structure of the dynamic motion is shown in Fig. 9. Using controller function, we can calculate the angular joint acceleration directly by referring to the latest state of the motion capture data input. After that, the simulators update the process through dynamic character motion. The previous result from this structure is the output angular acceleration as the sum of $\ddot{\theta}_{\text {initial }}$ and the difference of the angular acceleration $\Delta \ddot{\Theta}$ [26]. Meanwhile, the results input based on combination of a human body model and external physical input for the controller and the simulator [26].

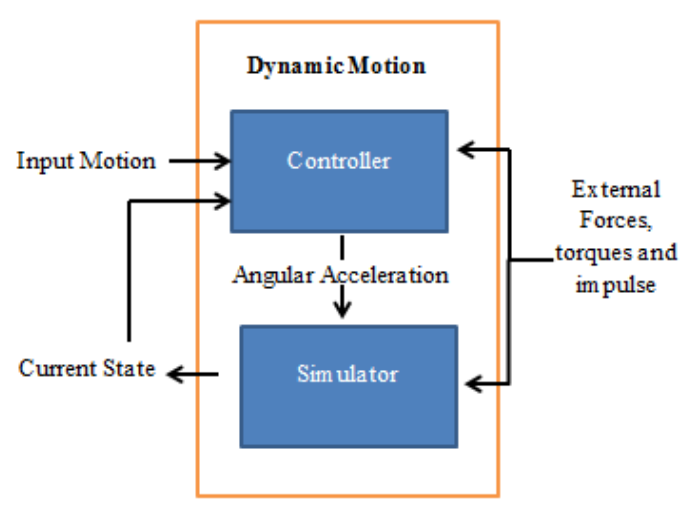

Fig. 9. Dynamic motion controller and simulator structure
Recent survey for the dynamic motion generation and control shows the new process of the virtual character motion control based simulation as shows in Fig. 10. This structure combined the active control torque and other external physical interaction. The output motions have been generated by the physical simulator. User needs to know to control the basic dynamic simulation of character movement. The methods linking the connection between forces acting to the body and acceleration can be categorized in two classes: maximal coordinate and reduced coordinate.

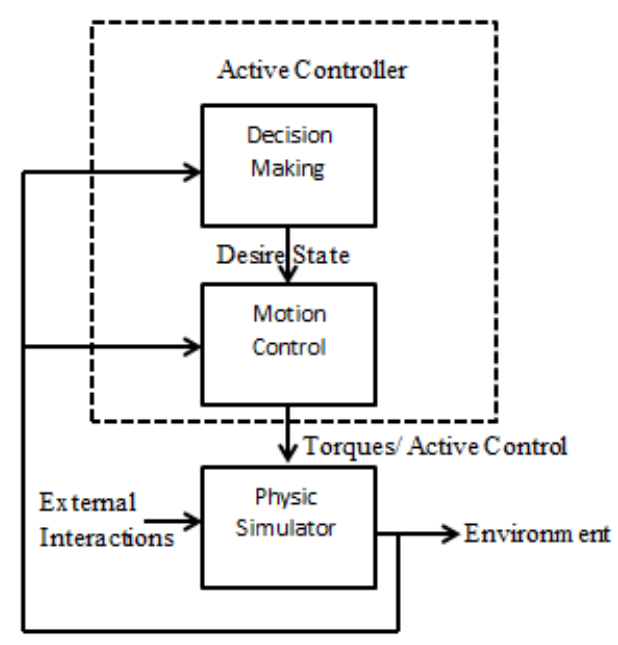

Fig. 10. Process of virtual character motion control based simulation [18] 


\section{CONCLUSION}

In this paper, a review of the main parts in dynamic 3D character motion in game development has been carried out. These main components can be classified into three categories as follows: (a) skeleton hierarchy and kinematics; (b) motion capture data animation; (c) active dynamic control. This review is very important to students and researchers in understanding the concepts and techniques of character motion in real time animation.

The main challenge for character motion in real time animation is to make the character move automatically and instructed like real human. Multiple learned models and different control methods need to be explored for the purpose of getting a natural, balanced dynamic character motion while maintaining the character's physical properties [28, 29]. Editing and modifying techniques for motion capture animation data need to be maximized to the highest level to achieve realistic and convincing motions for virtual characters.

\section{ACKNOWLEDGMENT}

This research was supported by UTM VicubeLab at Department of Computer Graphics and Multimedia, Faculty of Computer Science and Information System, Universiti Teknologi Malaysia. Special thanks to Universiti Teknologi Malaysia (UTM) Vot. 00J44 Research University Grant Scheme (RUGS) for providing financial support of this research.

\section{REFERENCES}

[1] Yueqi Hu, Shuangyuan Wu, Shihong Xia, Jinghua Fu, Wei Chen, et al. (2010). Motion track: Visualizing variations of human motion data. Pacific Visualization Symposium (PacificVis), 2010 IEEE.

[2] M. Oshita, (2006). Motion-capture-based avatar control framework in third-person view virtual environments. Proceedings of the 2006 ACM SIGCHI international conference on Advances in computer entertainment technology. Hollywood, California, ACM: 2.

[3] C. Kirmizibayrak, J. Honorio, X. Jiang, R. Mark and J.K. Hahn (2011). Digital analysis and visualization of swimming motion. In The International Journal of Virtual Reality, 2011, 10 (3), pp. 9-16.

[4] P. Wrotek, O. C. Jenkins, et al. (2006). Dynamo: dynamic, data-driven character control with adjustable balance. Proceedings of the 2006 ACM SIGGRAPH Symposium on Videogames. Boston, Massachusetts, ACM: 61-70.

[5] Z. Xiao, X. Yang, et al. (2005). Motion Data Correction and Extrapolation Using Physical Constraints. Proceedings of the Ninth International Conference on Information Visualisation, IEEE Computer Society: 571-576.

[6] D. Kim, M. Sung, et al. (2005). Realtime Control for Motion Creation of 3D Avatars. Advances in Mulitmedia Information Processing - PCM 2005. Y.-S. Ho and H. Kim, Springer Berlin / Heidelberg. 3767: 25-36.

[7] Tianyu Huang, Jia Yang, Lijie Li, et al. (2009). Data-driven online motion analysis. Computer-Aided Industrial Design \& Conceptual Design, 2009. CAID \& CD 2009. IEEE 10th International Conference on.

[8] A. Witkin, M. Kass, (1988) Motion Warping. In Computer Graphics. Proceedings of the 1988 SIGGRAPH: pp. 159-168.

[9] Z. Popovic, A.Witkin (1999) Physically Based Motion Transformation. Proceeding of the 1999 SIGGRAPH: pp.11-20.
[10] A. Safona, J. Hodghins, et al. (2004) Synthesizing Physically Realistic Human Motion in Low Dimensional, Behaviour- Specific Spaces. Proceeding of the $31^{\text {st }}$ Annual Conference on Computer Graphics and Interactive Techniques, ACM Press

[11] P. Szczuko, B. Kostek, et al. (2009). New Method for Personalization of Avatar Animation. Man-Machine Interactions. K. Cyran, S. Kozielski, J. Peters, U. Stanczyk and A. Wakulicz-Deja, Springer Berlin / Heidelberg. 59: 435-443.

[12] C. Woong, T. Ono, et al. (2009). Body Motion Analysis for Similarity Retrieval of Motion Data and Its Evaluation. Intelligent Information Hiding and Multimedia Signal Processing, 2009. IIH-MSP '09. Fifth International Conference on.

[13] M. Alexa and W. Müller (2000). "Representing Animations by Principal Components." Computer Graphics Forum 19(3): 411-418.

[14] Y. Abe, C. K. Liu, et al. (2004). Momentum-based parameterization of dynamic character motion. Proceedings of the 2004 ACM SIGGRAPH/Eurographics symposium on Computer animation. Grenoble, France, Eurographics Association: 173-182.

[15] X. Liang, Z. Wang, W. Geng and F. Multon (2011). A motion-based user interface for the control of virtual human perfoming sports. In The International Journal of Virtual Reality, 2011, 10 (3), pp. 1-8.

[16] C. F. Rose Iii, P.-P. J. Sloan, et al. (2001). Artist-Directed Inverse-Kinematics Using Radial Basis Function Interpolation. Computer Graphics Forum 20(3): 239-250.

[17] E. S. L. Ho, T. Komura, et al. (2010). Spatial relationship preserving character motion adaptation. ACM Trans. Graph. 29(4): 1-8.

[18] L. Shilei, L. Jiahong, et al. (2009). A Survey of Dynamic Motion Generation and Control for Virtual Characters. Computational Intelligence and Software Engineering, 2009. CiSE 2009. International Conference on

[19] C. Kwang-Jin and K. Hyeong-Seok (1999). On-line motion retargetting. Computer Graphics and Applications, 1999. Proceedings. Seventh Pacific Conference on.

[20] M. Gleicher (1998). Retargetting motion to new characters. Proceedings of the 25th annual conference on Computer graphics and interactive techniques, ACM, pp. 33-42.

[21] C. K. Liu, Z. Popovi, et al. (2002). Synthesis of complex dynamic character motion from simple animations. ACM Trans. Graph. 21(3): 408-416.

[22] N. I. Badler, M. S. Palmer, et al. (1999). Animation control for real-time virtual humans." Commun. ACM 42(8): 64-73.

[23] W. Pedryz, G F. omide, et al (2007) .Fuzzy System Engineering: Toward Human-Centric Computing, Wisley-IEEE Press, New Jersey.

[24] P. Sardain and G. Bessonnet (2004). Forces acting on a biped robot. Center of pressure-zero moment point. Systems, Man and Cybernetics, Part A: Systems and Humans, IEEE Transactions on 34(5): 630-637.

[25] K. Yamane and Y. Nakamura (2003). Dynamics Filter - concept and implementation of online motion Generator for human figures.Robotics and Automation, IEEE Transactions on 19(3): 421-432.

[26] M. Oshita and A. Makinouchi (2002). A dynamic motion control middleware for computer games. ACM SIGGRAPH 2002 conference abstracts and applications. San Antonio, Texas, ACM: 139-139.

[27] Hoshang Kolivand, Mohd Shahrizal Sunar Azam Amirshakarami and Zhila Ranjbar (2011). Real-Time Volume Shadow using Visible-Non Visible Algorithm. Journal of Computer Science, 7(7), pp: 980-985

[28] Ismahafezi Ismail, Mohd Shahrizal Sunar, Mohd Kufaisal Mohd Sidik and Cik Suhaimi Yusof(2011). A Review of Dynamic Motion Control Considering Physics for Real Time Animation Character. 2011 Workshop on Digital Media and Digital Content Management: pp. 86-90

[29] Mohd Kufaisal bin Mohd Sidik, Mohd Shahrizal bin Sunar, Ismahafezi bin Ismail, Mohd Khalid bin Mokhtar and Normal binti Mat Jusoh (2011). A Study on Natural Interaction for Human Body Motion Using Depth Image Data. 2011 Workshop on Digital Media and Digital Content Management: pp. 97-102 


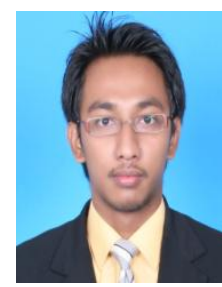

simulation

Ismahafezi Ismail is currently a $\mathrm{PhD}$ candidate in Computer Science at the Department of Computer Graphics and Multimedia, Universiti Teknologi Malaysia. He received the MSc in Computer Science (2009), and BE degree in Mechanical Engineering (2007) from Universiti Teknologi Malaysia. His research interest includes real-time computer graphics, computer animation and games, character motion, physic based animation, natural interaction, virtual reality and

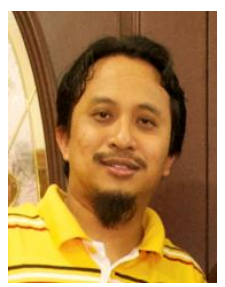

Mohd Shahrizal Sunar obtained his PhD from National University of Malaysia in 2008. His major field of study is real-time and interactive computer graphics and virtual environment. He received his MSc in Computer Graphics and Virtual Environment (2001) from The University of Hull, UK and BSc degree in Computer Science majoring in Computer Graphics (1999) from Universiti Teknologi Malaysia. He served as academic member at Computer Graphics and Multimedia Department, Faculty of Computer Science and Information System, Universiti Teknologi Malaysia since 1999. Since 2009, he had been given responsibility to lead the department. The current research program that he lead are Driving Simulator, Augmented Reality, Natural Interaction and Creative Content Technology. He had published numerous articles in international as well as national journals, conference proceedings and technical papers including article in magazines. Dr. Shahrizal is an active professional member of ACM SIGGRAPH. He is also a member Malaysian Society of Mathematics and Science.

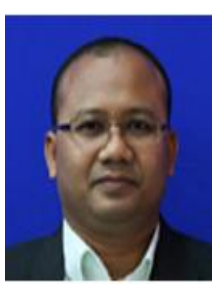

Cik Suhaimi Yusof received MSc in Computer Science majoring in Distributed Multimedia from University of Leeds in 1998. He later obtained the BSc degree in Computer education from Universiti Teknologi Malaysia on 1996. He is a faculty member at Department of Computer Graphics and Multimedia, Faculty of Computer Science and Information System, Universiti Teknologi Malaysia since 1996. Focus of his research in computer graphics modeling, data visualization and

augmented reality. 